Meta

Journal des tradlucteurs

Translators' Journal

\title{
Combinatoire, terminologies et textes
}

\section{Jacques Lethuillier}

Volume 36, numéro 1, mars 1991

La terminologie dans le monde : orientations et recherches

URI : https://id.erudit.org/iderudit/003504ar

Aller au sommaire du numéro

Éditeur(s)

Les Presses de l'Université de Montréal

ISSN

0026-0452 (imprimé)

Découvrir la revue

Citer cet article

Lethuillier, J. (1991). Combinatoire, terminologies et textes. Meta, 36(1), 92-100.

Ce document est protégé par la loi sur le droit d'auteur. L'utilisation des services d'Érudit (y compris la reproduction) est assujettie à sa politique d'utilisation que vous pouvez consulter en ligne.

https://apropos.erudit.org/fr/usagers/politique-dutilisation/
Cet article est diffusé et préservé par Érudit.

Érudit est un consortium interuniversitaire sans but lucratif composé de l’Université de Montréal, l’Université Laval et l’Université du Québec à Montréal. Il a pour mission la promotion et la valorisation de la recherche. https://www.erudit.org/fr/ 


\section{COMBINATOIRE, TERMINOLOGIES ET TEXTES}

JACQUES LETHUILLIER

Université de Montréal, Montréal, Canada

Il n'est pas un colloque, pas un événement centré sur la traduction ou la terminologie où la question de l'intérêt présenté par la cooccurrence en langues de spécialité ne soit soulevée. L'observation que l'on fait couramment est que les outils décrivant la façon d'utiliser les mots en discours, le comportement syntagmatique des termes, sont rares. Et s'en suivent généralement des descriptions de ce que devraient contenir de tels outils pour être efficaces.

Par ailleurs la consigne semble être donnée aux terminologues d'exclure de leur liste de repérage toute unité qui ne serait pas un substantif. Aussi, on oppose régulièrement lexicographie et terminologie sur la base du fait que la première part du texte dans sa démarche (sémasiologique) pour associer des sens aux mots, tandis que la seconde pose en principe l'indépendance des objets de son traitement par rapport aux textes.

On peut penser voir une contradiction entre l'affirmation de tels besoins par les utilisateurs des langues de spécialité, d'une part, et les affirmations réitérées des linguistes qui se sont efforcés d'asseoir l'activité terminologique sur des bases théoriques.

Des liens variés et forts existent entre terminologie et texte. La terminologie emprunte aux textes, puise dans les textes. Elle est aussi prise dans le texte. Et cela peut vouloir dire qu'elle est toute dans les textes ou qu'elle utilise la forme du texte comme moyen d'expression. À l'inverse, le texte, qui réexprime la terminologie dans sa composante descriptive, peut être vu comme le résultat de la mise en œuvre d'une combinatoire fortement contrainte par la terminologie. Terminologie et texte sont indissociables, inextricablement liés même.

Dans un premier temps, nous essayons de dégager les liens qui existent entre terminologie et texte au plan abstrait. Nous voyons ensuite ce qui peut en être retiré au plan concret, soit de l'activité terminologique pratique, soit de l'activité de production d'énoncés, d'élaboration de textes.

Cet effort de réflexion sur certains aspects du fonctionnement de la terminologie et des langues de spécialité, paraîtra futile, dans la mesure où il se ramène à décrire des mécanismes qui correspondent à des automatismes pour chacun d'entre nous. Le fait que nous les mettions en æuvre très régulièrement de façon réflexe est toutefois susceptible d'avoir l'effet d'un masque et de nous empêcher d'aller plus loin, aussi loin que ce serait souhaitable pour certaines analyses. Par ailleurs, compte tenu de la difficulté de modéliser des systèmes comme les terminologies, les tentatives dans ce sens auraient intérêt à partir de champs où l'on observe le plus grand nombre de régularités. Ainsi il serait dans l'ordre des choses d'explorer les terminologies scientifiques et techniques avant de s'attaquer à l'analyse des systèmes représentés par la terminologie juridique ou les terminologies des sciences sociales, par exemple. Le recours à des logiques autres que la logique classique binaire pourrait se révéler nécessaire pour les appréhender. 


\section{AU PLAN ABSTRAIT}

\section{UNE FIN COMMUNE: LA DESCRIPTION}

Texte et terminologie partagent un objectif de communication, qui est l'objectif de description des choses.

La terminologie est à voir comme un système de description et de classement des objets individuels. Ou bien encore, selon un autre point de vue, comme l'activité ayant pour objet la description des notions, ainsi que le recensement et l'étude des formes linguistiques servant à les désigner, selon une démarche largement sémasiologique - les notions étant des caractères généralisés dont l'ensemble forme le puissant instrument de description et de classement que l'on vient d'évoquer. La terminologie décrit les objets individuels à travers les notions.

Le texte comporte une composante descriptive souvent importante, mais qui peut déborder largement la terminologie de plusieurs façons. Pour une part de cette composante, il constitue une redondance par rapport à la terminologie qu'il réexprime. Par ailleurs, le texte évoque des situations en précisant des liens moins forts, moins permanents entre les objets. En outre, il sort de la terminologie en surdéterminant les notions les plus spécifiques.

\section{L'IDÉE DE RELATION: AU CCEUR DU PROCESSUS DE DESCRIPTION}

Si leurs fins en matière de description les différencient, terminologie et texte partagent le recours au même mécanisme de base, centré sur l'idée de relation, en rapport direct avec la notion de combinatoire.

Les idées de relation servent à rattacher les uns aux autres des objets individuels ou des groupes d'objets individuels, par quelque aspect susceptible de les caractériser. De façon générale elles sont à la base de toute description. Pour décrire les objets individuels du type artefact ou objet technique, par exemple, il existe un certain nombre d'idées de relation primitives sur lesquelles sont construites l'ensemble des idées de relation. Ce sont notamment les idées de contiguîté dans l'espace, de relation de cause à effet, de rapport matière - produit, etc.

Ainsi:

- pied est en relation de partie à tout avec jambe. Le pied compose la jambe. La relation inverse serait exprimée par une jambe comporte un pied. De la même façon, pied est en relation de contenu à contenant avec soulier. Ici encore la relation est polarisée.

- arbre et terre sont en rapport de contiguïté dans l'espace. Il s'agit d'une relation réciproque.

- juin et juillet sont en rapport de contiguïté dans le temps.

Une idée de relation possède deux paramètres, qui sont des termes évoquant les classes dont les objets particuliers entretiennent les uns avec les autres cette relation. Si l'on voit la relation à partir de l'une des classes d'objets servant de paramètres, on retrouve la structure d'un prédicat. Une classe forme le thème (arbre, par exemple ici). La deuxième classe (terre, ici) joue le rôle d'argument. Elle précise la portée de la relation, et, avec cette dernière, définit un prédicat, qui est un caractère ou attribut que possède les objets de la classe thème. Le verbe servant à exprimer la relation constitue le noyau ou base du prédicat.

\section{MÉCANISME DE BASE DE LA DESCRIPTION}

Plusieurs caractères indépendants forment un schéma de description. S'il est retenu par la terminologie, on parle de notion terminologique. Un tel schéma joue en quelque 
sorte le rôle de filtre conceptuel à objets individuels. Ceux qui sont conformes au schéma, qui présentent les caractères passent à travers le filtre, les autres sont arrêtés.

Le processus de description des objets particuliers ou particularisation se fait sur l'axe horizontal, par ajout successif de caractères indépendants pour enrichir un schéma donné. Cela constitue un premier plan. Mais les descriptions d'objets ne s'arrêtent pas nécessairement à un premier plan. Elles peuvent comporter un nombre variable plus ou moins grand de niveaux, pour chaque caractère indépendant, qui définit en quelque sorte une dimension d'exploration ou d'analyse.

La particularisation se fait alors suivant l'axe vertical, en profondeur, selon une démarche récurrente, un caractère indépendant donné pouvant être déterminé plusieurs fois de suite. La détermination vise la relation elle-même ou les objets avec lesquels celle-ci est entretenue. $\mathrm{Vu}$ depuis un schéma spécifique qu'il a permis de construire à partir d'un schéma plus général, le caractère apparaît comme un moyen de détermination. Vu depuis le schéma spécifique dont il fait partie, dans la perspective de construire des schémas plus détaillés encore, il apparaît comme une indétermination.

Une manière encore plus simple de présenter les choses consiste à dire qu'un caractère représente une imprécision si l'on est pessimiste et une précision si l'on est optimiste. En tant qu'imprécision ou indétermination, le caractère représente une palette descriptive, assimilable à une série de virtualités. En d'autres mots il s'agit d'une série de possibilités de réalisation mutuellement exclusives, c'est-à-dire dont une seule à la fois peut s'actualiser dans un objet individuel.

Deux mécanismes sont mis en œuvre pour faire jouer son rôle de palette descriptive à un caractère, qui peut devenir un véritable arbre de particularisation: soit la spécialisation récurrente de la relation; soit la limitation de sa portée par éclatement de la classe que définit le terme jouant le rôle d'argument.

Prenons le cas des objets voitures automobiles. Ces objets sont dans le rapport tout partie avec moteur, caractère exprimable par la proposition «les voitures automobiles comportent un moteur». Une telle proposition, qui réexprime le système terminologique est interprétable comme une imprécision.

Imprécision quant à la nature du rapport liant l'objet et sa composante moteur, d'abord. Le moteur peut être placé à l'avant du tout (la voiture), à l'arrière ou au centre. Par ailleurs, dans chacun de ces cas, il peut être disposé de façon verticale, horizontale ou inclinée. Cela donne le tableau à la page suivante. 


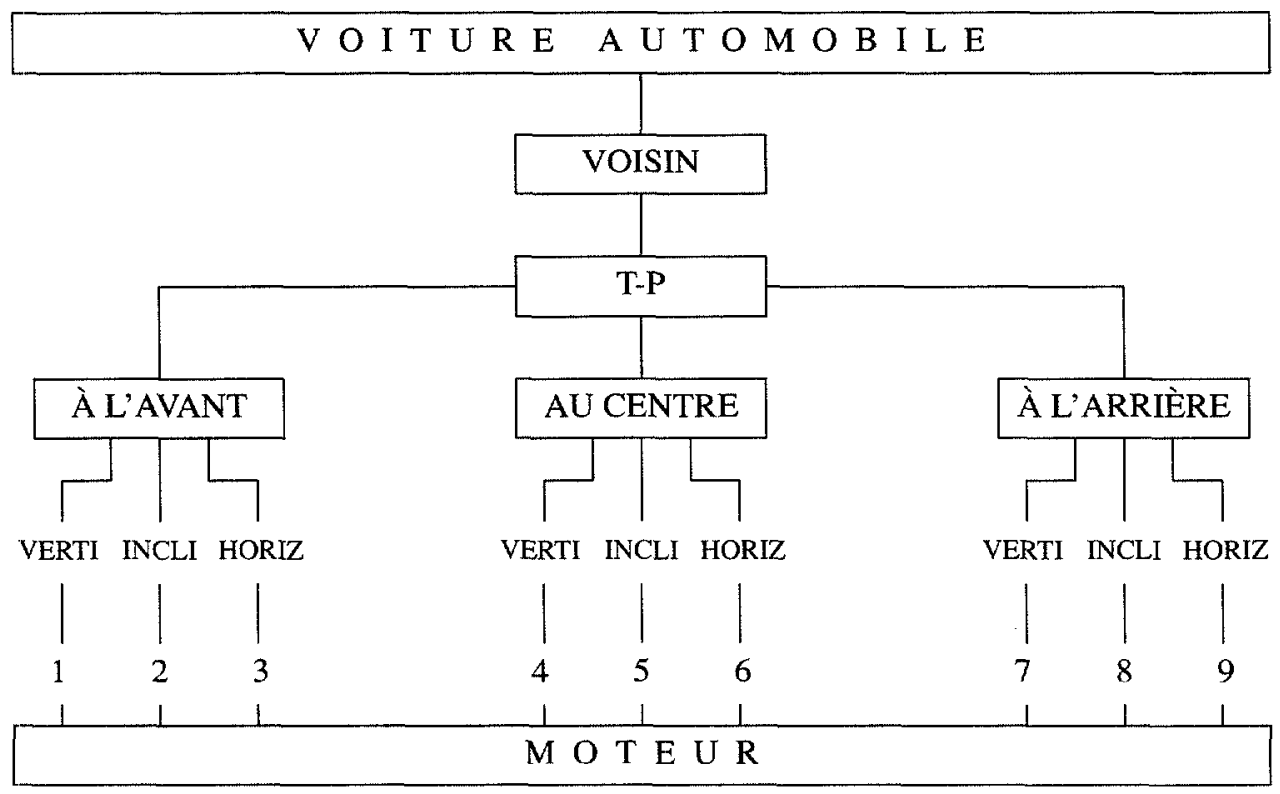

L'idée de relation tout-partie, qui spécialise celle de contiguïté dans l'espace, se trouve particularisée deux fois de suite, et cela conduit finalement à neuf types de rapports différents des objets voiture automobile avec les objets moteur.

On pourrait maintenant, voir chacune de ces valeurs de la relation tout-partie, qui détermine en fait un sous-ensemble dans le grand ensemble voiture automobile, comme l'élément constant d'un caractère dont le terme variable serait cette fois-ci représenté par l'ensemble des objets avec lequel la relation existe. Ici l'ensemble évoqué par la classe terminologique moteur. En quelque sorte, la portée de la relation, et non plus sa nature, serait limitée par éclatement de l'ensemble moteur en sous-classes et restriction à une sous-classe. Pour rester simple, on pourrait avoir l'espace moteur divisé en deux classes exclusives: celle des moteurs suralimentés et celle des moteurs atmosphériques (non suralimentés). Finalement, en jouant sur les deux composantes du caractère, on obtiendrait une palette de dix-huit valeurs.

\section{L'ARTICULATION TERMINOLOGIE-TEXTE}

La terminologie emprunte au texte pour l'expression de ses schémas propres. Elle utilise le mécanisme fondamental de la production d'énoncés élémentaires à cette fin: la combinatoire. Elle utilise aussi les idées de relation offertes par la langue pour construire ces schémas, dont certains appartiennent à la terminologie.

Par ailleurs le texte emprunte à la terminologie ses schémas construits, qui constituent une sorte de raccourci pour aller au-delà dans le processus de particularisation des objets ou pour décrire des situations.

La terminologie construit un espace conceptuel discret, borné, dans lequel sont projetés les référents. Il est borné de deux façons. Par la volonté des spécialistes, qui fixent conventionnellement des limites à la fonction remplie par la terminologie, en premier lieu; et par la singularité du monde des référents, qui forment des groupes entre lesquels existent des vides. 
Un mécanisme fondamental de la terminologie est l'héritage dans le processus de spécialisation, qui s'accompagne de la valuation de caractères «pendants». Quand on passe d'une classe terminologique générale à une sous-classe spécifique, par «forçage» d'un caractère (dit pertinent ou classificateur) à une valeur, cela peut avoir pour effet de déterminer un ou plusieurs autres caractères pendants, pas totalement indépendants, les valeurs prises par ces caractères étant qualifiées de caractères consécutifs. Qui plus est, un tel caractère, dans le même processus de particularisation, peut se trouver «lié» plusieurs fois de suite.

L'ensemble de ces caractères constitue la surcharge sémantique, c'est-à-dire l'écart entre la somme de la notion générique et du caractère pertinent, d'une part, et la notion spécifique, d'autre part. Mais il y a plus intéressant à observer à ce sujet. C'est le fait que sur une dimension, plusieurs palettes successives se trouvent alors verrouillées dans le sous-espace conceptuel qui part de la classe considérée. Des places sont inoccupées dans l'espace de représentation mentale offert par la terminologie, qui correspondent à ce que l'on pourrait appeler des vides référentiels. Ils représentent des contraintes à satisfaire dans la production d'énoncés valides.

Le texte repousse les limites inscrites dans la terminologie. Il précise des relations restées à l'état de caractères pendants dans les schémas de description les plus spécialisés offerts par la terminologie, et évocables de façon extrêmement concise par un terme. Dans ce sens le texte permet une sorte de surdétermination par rapport à la terminologie, se situant entre le degré de détermination maximal des notions les plus spécifiques et la détermination intégrale implicite qui se trouve attachée aux objets individuels. Le texte occupe les vides terminologiques (et ce serait plus juste de dire sort de la terminologie), mais pas les vides référentiels.

\section{AU PLAN CONCRET}

\section{DE L'ACTIVITÉ TERMINOLOGIQUE émergence des concepts}

Les notions préexistent à l'activité terminologique, dans l'esprit des spécialistes d'abord, même si c'est de façon encore floue, puis dans leurs écrits qui servent de ponts, mais sous une forme diffuse. La description des notions faite par les spécialistes est fragmentaire et dispersée dans les textes. À l'occasion de l'émergence d'un concept, les efforts menés en vue de lui associer un nom, en même temps qu'on tente de le définir, de le décrire s'opèrent dans une certaine mouvance. Cette activité initiale, qui procède d'une démarche à la fois onomasiologique et sémasiologique, échappe au terminologue professionnel, dont l'intervention moins noble se situe en aval.

La stabilisation n'intervient qu'après des échanges d'arguments à l'occasion d'événements comme des colloques ou des congrès, qui le plus souvent se retrouvent sous la forme d'écrits. Il s'agit de descriptions fragmentaires, inachevées, qui, depuis le niveau le plus élevé des spécialistes où elles ont été produites originellement, diffusent vers le bas jusqu'au niveau de la vulgarisation et de la didactique, en se simplifiant ou en s'altérant.

\section{exploitation des textes}

Dans la phase qui suit le repérage, mais qui précède la description des notions, le terminologue accumule les matériaux. Il les puise presque exclusivement dans les textes, qu'il choisit pour l'importance de leur composante descriptive.

La matière intéressante des textes de LS correspond en fait à la partie descriptive où se trouvent exprimées des relations permanentes existant entre les objets. Il s'agit d'un discours terminologique noyé dans des textes à vocation non terminologique. 
Dans une perspective d'automatisation de la tâche qui consiste à réunir des prédicats utiles pour la description des notions dont on a pu faire une liste préalablement, il serait souhaitable d'analyser les particularités de cette composante des textes et notamment d'examiner dans quelle mesure elle ne se distingue pas par la présence de marqueurs spécifiques. La direction dans laquelle il conviendrait de chercher est en rapport avec une particularité nette de cette composante termino-encyclopédique des textes de LS, leur caractère de redondance découlant du fait qu'elle réexprime un contenu normalement considéré comme évoqué implicitement par le terme.

La tâche fastidieuse de collecte des données, préparation incontournable au travail terminologique proprement dit, de reconstruction, constitue pour l'instant encore un véritable goulet d'étranglement. Son informatisation, même partielle, permettrait un déblocage. Mais pour faire exécuter à un logiciel une sélection intelligente des contextes dans un corpus électronique, sur la base de la reconnaissance de marqueurs spécifiques, il faudra formaliser ces marqueurs.

\section{DE LA PRODUCTION D'ÉNONCÉS}

Il existe un autre point de vue, radicalement opposé, sous lequel on peut examiner les rapports qui unissent terminologie et texte-combinatoire. Celui de l'élaboration des textes, de la production d'énoncés.

\section{texte et combinatoire}

Le texte est un enchaînement, selon une logique en rapport avec un objectif de communication, d'énoncés élémentaires qui sont des prédicats représentant des choses que l'on dit à propos d'autres choses. D'un point de vue purement descriptif, ces énoncés élémentaires prennent la forme de combinaisons d'unités appartenant à des catégories différentes de la langue. En langue de spécialité, une catégorie est fortement représentée dans les combinaisons: c'est le terme. Par ailleurs la combinaison véritablement productive est de la forme SUBSTANTIF (terme) - VERBE - SUBSTANTIF (terme).

L'élaboration de textes repose sur la production de combinaisons de ce type. Cela revient à exploiter deux ressources centrales des langues de spécialité - le vocabulaire notionnel ou descriptif (les termes) et le vocabulaire de fonctionnement (essentiellement formé par les verbes, exprimant des idées de relations).

L'art de produire des combinaisons, de réaliser cette intégration, est appelée combinatoire. C'est le mécanisme fondamental sur lequel repose la production d'énoncés élémentaires. D'où l'intérêt d'essayer de le comprendre, notamment pour les utilisateurs d'une langue de spécialité dont la fonction est d'élaborer des textes.

\section{une terminologie redondante}

Une terminologie redondante s'est établie autour de cet aspect essentiel de la langue commune et des langues de spécialité qu'est la combinatoire. De façon générale, pour une langue de spécialité, la combinatoire est l'aspect en rapport avec les combinaisons d'unités que l'on observe dans les textes ou qu'elle permet de produire. Une combinaison est l'association, sur l'axe syntagmatique d'unités appartenant à des catégories différentes ou à la même catégorie (termes, alors). Le mot syntagme est employé dans le même sens.

Pour exprimer le fait que différentes unités apparaissent ensemble dans la chaîne parlée, on dit qu'il y a cooccurrence ou collocation de ces unités. Si la cooccurrence est vue à partir d'une unité privilégiée (le verbe, par exemple), la tendance sera de parler de son environnement syntagmatique pour désigner l'ensemble des autres unités apparaissant avec elle dans la combinaison ou syntagme. Le terme «comportement syntagmatique» s'emploie dans le cas où l'on pense, à propos d'une unité donnée, plus à 
une règle permettant de prévoir quels en sont les cooccurrents possibles qu'à la liste de ceux-ci.

Dans le cas de la combinatoire verbale (centrée sur le verbe), qui nous intéresse ici, les cooccurrents ou collocateurs du verbe sont le thème et le ou les arguments du prédicat, qui peuvent être vus fonctionnellement comme des actants. Le verbe, exprimant l'idée de relation est la base de la cooccurrence, et en même temps le noyau du prédicat.

\section{description de la combinatoire}

La combinatoire étant le mécanisme fondamental de la production d'énoncés élémentaires, il serait souhaitable, pour les utilisateurs d'une langue de spécialité qu'il en existe une description sous une forme ou une autre.

En première approximation, définie sans nuance, la combinatoire est l'ensemble des combinaisons exprimant des relations entre paires de référents ou de classes de référents qui sont possibles ou non aberrantes.

Idéalement les utilisateurs non spécialistes d'une langue de spécialité aimeraient disposer d'outils d'aide à la rédaction qui recensent les combinaisons possibles. Malheureusement, il serait tout à fait irréaliste de vouloir proposer une description en extension de la combinatoire pour une langue de spécialité.

On peut voir aussi la combinatoire comme l'ensemble des règles régissant la production des combinaisons possibles, autrement dit des règles définissant le comportement syntagmatique des verbes ou des termes, la façon dont ils s'associent les uns aux autres, essentiellement au plan de la logique du système notionnel. Mais chercher à préciser pour chaque verbe, par exemple, ou chaque substantif, des règles qui en fixeraient l'emploi en discours et constitueraient donc une description en compréhension de la combinatoire risquerait de se révéler aussi utopique.

La combinatoire, c'est encore, si l'on change dc point de vue, l'ensemble des combinaisons non permises. Parler de combinaisons non permises, cela revient à dire qu'on ne peut tenir, relativement à des objets référencés par un terme évoquant implicitement un schéma de description, n'importe quel propos. Une description de la combinatoire ainsi perçue, en extension aussi bien qu'en compréhension, sous la forme de règles d'exclusion, ne paraît a priori pas plus accessible. Ce point de vue n'est pas néanmoins complètement dénué d'intérêt dans la mesure où il nous amène à réfléchir sur le fait que si des combinaisons sont impossibles, c'est qu'il existe un système de contraintes à l'origine de tels interdits ou exclusions.

La connaissance de la nature de ces contraintes et de la façon dont elles opèrent pourrait nous aider à contourner le problème de la description de la combinatoire. Il s'agirait en quelque sorte de découvrir l'origine de telles contraintes et de chercher si elles ne pourraient pas s'exprimer sous la forme de métarègles à partir desquelles le comportement syntagmatique des verbes ou des substantifs se déduirait.

L'aisance avec laquelle les utilisateurs confirmés d'une LS produisent des combinaisons valides laisse penser en effet que la combinatoire est réductible à un modèle simple formé de quelques métarègles que le spécialiste applique inconsciemment, sans faire d'effort. Dans une perspective de formation, s'efforcer d'analyser et d'expliquer le fonctionnement de la combinatoire à ce niveau très général serait certainement du plus haut intérêt. Le choix d'une méthode de traitement pour l'élaboration d'outils d'aide à la rédaction centrés sur la combinatoire devrait aussi s'appuyer sur une réflexion dirigée dans ce sens. Une description aussi générale pourrait être qualifiée de métacombinatoire. 


\section{métacombinatoire}

Nous postulons l'existence d'une métacombinatoire, qui n'est rien d'autre que la description au niveau le plus général, donc en termes très abstraits, des contraintes qu'exercent sur le texte l'un des constituants des énoncés élémentaires, à savoir le terme et ce à quoi il renvoie. D'une certaine façon, faire une telle hypothèse, c'est reconnaître que le texte peut tout dire, mais que, en même temps, la terminologie et le monde des référents, des objets individuels, fixent une limite aux choses dites valides.

D'une part, les prédicats doivent exprimer des relations avec le reste de l'univers qui ont une substance, autrement dit qui ne tombent pas dans un vide référentiel. D'autre part, ils ne doivent pas être en contradiction avec le découpage du monde des référents opéré par la terminologie.

Parler de vides référentiels, c'est rappeler que le monde des référents, des objets individuels, est extrêmement singulier. Projeté dans l'espace de représentation mentale ou espace conceptuel offert par la terminologie, il n'en occupe qu'une faible partie. Nous pourrions donner des exemples simples illustrant cette affirmation insolite. Celui de réfrigérateur qui évoque des objets dont la forme est toujours parallélépipédique pour des raisons évidentes. Le caractère forme, qui en fait traduit un rapport de contiguilté dans l'espace entre ces objets et l'extérieur (leurs limites), est très déterminé et nous sommes donc très bas dans l'arbre de particularisation qui correspond à cette dimension de la description.

Comme autre exemple, on pourrait citer les cuves de réacteurs nucléaires de fission qui sont toujours cylindriques, pour des raisons moins simples que dans le cas des réfrigérateurs, mais tout aussi nettes, en rapport avec l'optimisation de la réaction au plan du bilan neutronique. En fait des centaines d'exemples sont à notre portée quand on y réfléchit. Et l'on peut les chercher aussi bien dans le monde des objets techniques que dans celui des objets naturels.

Par ailleurs, chaque fois que l'on descend dans l'arbre de particularisation, c'est-àdire que l'on enrichit le schéma de description des objets individuels en forçant à une valeur un caractère - hérité ou nouvellement impliqué - pour créer une opposition et fonder une sous-classe, il y a surcharge sémantique: d'autres caractères pendants prennent une valeur, ce qui a pour effet de «barrer» en quelque sorte les autres possibilités de réalisation pour ces caractères. À mesure que progresse la détermination et que donc l'extension des classes diminue, le nombre des palettes descriptives barrées augmente. Il s'ensuit que dans le texte, si l'on évoque un objet individuel par un terme se plaçant à un certain niveau de spécificité, associé à un actualisateur, ou par un terme plus général qui renvoie par le contexte au schéma de description correspondant à ce terme spécifique, toutes les relations verrouillées des palettes par lesquelles on est passé en descendant dans l'arbre de particularisation sont interdites. Cette deuxième contrainte peut se résumer en disant que le texte ne doit pas être en contradiction avec l'implicite de la terminologie.

\section{combinatoire logique et combinatoire d'usage}

À ce stade il reste à dissiper une confusion à propos de l'idée vague de combinatoire qui se dégage des écrits actuels. La combinatoire dont on parle habituellement n'est pas du tout celle dont il a été question dans cet article, et que l'on pourrait qualifier de combinatoire logique.

Â côté de cette combinatoire logique, il en existe une autre, et peut-être la seule qui soit intéressante d'un point de vue pratique, à laquelle on devrait donner le nom de combinatoire d'usage [combinatoire lexicale restreinte pour Igor Mel'čuk (Mel'cuk 1984 : 4)]. Très difficilement réductible à un schéma, elle se présente sous la forme d'un 
système de surcontraintes se greffant sur la combinatoire logique plus facile à appréhender.

Dans le cas de la combinatoire verbale, par exemple, chaque fois qu'une idée de relation est susceptible d'être exprimée valablement par plusieurs unités de la langue et que l'emploi de l'une (ou de certaines) d'entre elles est très fortement privilégié par l'usage au point de rendre insolite l'emploi des autres unités concurrentes, il convient de parler de combinatoire d'usage. Le recours à la métaphore pour exprimer des idées de relation s'inscrit presque automatiquement dans la combinatoire d'usage, du fait de la rupture que cela crée. L'analyse de ce type de combinatoire semble a priori beaucoup plus difficile que celui de la combinatoire logique. On voit mal qu'un traitement autre qu'une description en extension lui soit appliqué. La difficulté d'un tel travail serait alors lié à sa reconnaissance et à l'élimination de la redondance.

L'intérêt d'étudier le fonctionnement des langues de spécialité aux différents points de vue évoqués ci-dessus n'est pas purement théorique. Dans le contexte de la traduction spécialisée, et plus spécialement de la formation, le fait de pouvoir expliquer, par exemple, comment les contraintes de la combinatoire, découlant de l'organisation du système notionnel, permettent de lever des ambiguïtés du texte de départ, revêt la plus grande importance. Et l'on peut imaginer sans peine que l'automatisation de la traduction dans les domaines spécialisés ne serait pas envisageable sans l'encodage de règles traduisant de telles contraintes.

Il serait aussi du plus haut intérêt d'en arriver à proposer une description formalisée de la façon dont les rédacteurs spécialisés réalisent le marquage de certains segments de texte pour leur faire remplir une fonction spécifique ressortissant à la description des notions. Des recherches menées dans ce sens auraient des retombées au chapitre de l'enseignement de la terminologie et des langues de spécialité. Par ailleurs, l'informatisation de certaines activités terminologiques chronophages, notamment la sélection automatique de contextes utiles dans un corpus électronique, ne saurait se faire sans que l'on définisse au préalable des critères de reconnaissance d'un tel marquage.

\section{BIBLIOGRAPHIE}

HEID, Ulrich (1989): «Attente des terminologues à l'égard des programmes d'élaboration des dictionnaires», C. de Schaetzen (réd.), Terminologie diachronique, Paris, Conseil international de la langue française, pp. $221-242$.

KOCOUREK, Rostislav (1982): La langue française de la technique et de la science, Wiesbaden, Brandstetter, $259 \mathrm{p}$.

MEL'ČUK, Igor (1984): Dictionnaire explicatif et combinatoire du français comtemporain. Recherches lexicosémantiques I, Montréal, Les Presses de l'Université de Montréal, xvi + $172 \mathrm{p}$.

QUEMADA, Bernard (1978): «Technique et langage», Histoire des techniques, Paris, Encyclopédie de la Pléiade, Gallimard, pp. 1146-1240. 